\title{
Expanded genetic screening panel for the Ashkenazi Jewish population
}

\author{
Brett Baskovich, MD1, Susan Hiraki, MS, MPH², Kinnari Upadhyay, MS², \\ Philip Meyer², Shai Carmi, PhD³, Nir Barzilai, MD², Ariel Darvasi, PhD, Laurie Ozelius, PhD ${ }^{5}$, \\ Inga Peter, $\mathrm{PhD}^{5}$, Judy $\mathrm{H}$. Cho, $\mathrm{MD}^{5}$, Gil Atzmon, $\mathrm{PhD}^{2,6}$, Lorraine Clark, $\mathrm{PhD}^{7}$, Jin Yu, $\mathrm{PhD}^{8}$, \\ Todd Lencz, PhD ${ }^{8}$, Itsik Pe'er, PhD ${ }^{9}$, Harry Ostrer, $\mathrm{MD}^{1,2}$, and Carole Oddoux, $\mathrm{PhD}^{1,2}$
}

\begin{abstract}
Purpose: Carrier screening programs that identify the presence of known mutations have been effective for reducing the incidence of autosomal recessive conditions in the Ashkenazi Jewish (AJ) population and other populations. Yet, these programs have not realized their full potential. Furthermore, many known autosomal recessive and dominant conditions are not screened for and the molecular basis of other conditions for which screening might be offered is unknown.
\end{abstract}

Methods: Through literature review and annotation of full sequenced genomes from healthy individuals, we expanded the list of mutations. Mutations were identified in a sample of 128 fully sequenced $\mathrm{AJ}$ genomes that were filtered through clinical databases and curated manually for clinical validity and utility using the
American College of Medical Genetics and Genomics scoring (ACMG) system. Other known mutations were identified through literature review.

Results: A panel of 163 mutations was identified for 76 autosomal recessive, 24 autosomal dominant, and $3 \mathrm{X}$-linked disorders.

Conclusion: Screening for a broader range of disorders not only could further reduce the incidence of autosomal recessive disorders but also could offer the benefits of early or presymptomatic diagnosis.

Genet Med advance online publication 3 September 2015

Key Words: Ashkenazi Jews; carrier screening; genetic testing; variant annotation; whole genome sequencing

\section{INTRODUCTION}

Population-based genetic screening is a valuable application of genomic technology that can have significant clinical and public health benefits. Presymptomatic detection of disease and identification of increased disease risk provide the opportunity for early diagnosis, intervention, or prevention. Identification of genetic carrier status provides the opportunity for reproductive counseling and family planning, allowing for the prevention of the birth of an affected child or for early diagnosis and intervention. ${ }^{1}$ Many population-based screening programs target specific populations, such as newborns, particular ethnic groups, or those deemed to be at increased risk for certain diseases. With the advancement of genetic technologies, such as multiplex testing to conduct high-throughput genotyping of a multitude of variants, there has been an expansion in the number of diseases that can be screened for in the predisposition, presymptomatic, and carrier states. Selecting conditions to include on a population-based screening panel and even selecting the criteria to make this decision are still active questions.

Founder effects, in combination with endogamy and possible selection, have made some Mendelian conditions more prevalent in the Ashkenazi Jewish (AJ) population. Ethnicitybased carrier screening programs in this and other populations have been successful in decreasing the incidence of autosomal recessive conditions. ${ }^{1}$ The best-known example is Tay-Sachs disease, a severe, progressive neurodegenerative disorder caused by a defect in hexosaminidase A enzyme activity. Beginning in 1970, Tay-Sachs carrier screening in AJ individuals was the first population-based public health initiative aimed at decreasing the incidence of a lethal genetic disease. This coordinated effort, which included education, genetic counseling, and voluntary screening, was adopted throughout the United States as well as internationally. Within 30 years of its inception, the program was successful in decreasing the incidence of Tay-Sachs disease by more than $90 \%$ in the AJ populations of the United States and Canada. ${ }^{1}$ In addition to Tay-Sachs disease, there are many other recessive disorders that occur more commonly in the AJ population, and many laboratories now offer genetic carrier panels aimed at those of AJ ethnicity. The American College of Medical Genetics and Genomics (ACMG) and the American College of Obstetricians and Gynecologists have set forth recommendations for conditions that should be included

B.B. is the first author. S.H. and K.U. are co-first authors.

${ }^{1}$ Department of Pathology, Montefiore Medical Center, New York, New York, USA; ${ }^{2}$ Department of Pathology, Albert Einstein College of Medicine of Yeshiva University, New York, New York, USA; ${ }^{3}$ The Faculty of Medicine, Braun School of Public Health, Hebrew University of Jerusalem, Jerusalem, Israel; ${ }^{4}$ Department of Genetics, Hebrew University of

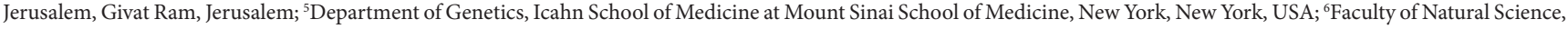
University of Haifa, Haifa, Israel; ${ }^{7}$ Department of Pathology, Columbia University, New York, New York, USA; ${ }^{8}$ The Feinstein Institute for Medical Research, Manhasset, New York, USA; ${ }^{9}$ Department of Computer Science, Columbia University, New York, New York, USA. Correspondence: Harry Ostrer (harry.ostrer@einstein.yu.edu) 
on an AJ panel. ${ }^{2}$ Despite these recommendations, there are differences between what is recommended and what is offered by laboratories, as well as differences among the various laboratories. In a multi-ethnic study of screening 23,000 individuals for $400+$ Mendelian variants, including variants found among $\mathrm{AJ}$, this ethnic group was the most likely to have carriers of serious recessive disorders. Many of these disorders were not included on the ACMG or American College of Obstetricians and Gynecologists lists of recommendations. ${ }^{2}$ In addition, the AJ community has generally been supportive of carrier screening for severe disorders and expansion beyond what is currently recommended. Therefore, expanding current AJ screening panels to include more conditions appears justified.

In addition to carrier screening panels, there are a growing number of predisposition genetic screening panels that identify those at increased risk for future disease. Currently, the targeted population tends to be those already deemed to be at increased risk due to their family history of disease. Cancer risk assessment represents an application of predisposition screening that provides the opportunity for prevention and early diagnosis to decrease risk. ${ }^{3}$ One such example is $B R C A 1 / 2$ screening, which serves as a paradigm for high-risk, population-based screening. With an increasing number of disease risk variants being discovered, genetic risk assessment for adult-onset conditions will continue to grow. ${ }^{4}$ Some who might benefit from genetic risk assessment may escape attention due to incomplete penetrance, sex-limited expression, and lack of or limited personal and/ or family history. ${ }^{5}$ Extending predisposition screening beyond those who meet standard high-risk criteria should have clinical utility, especially when offered in multiplex format.

As the number of disease variants grows and technological advances improve ease and efficiency of detection, criteria are needed against which to judge the merits of adding new genetic tests. In 1968, Wilson and Jungner delineated the principles of population-based screening, which require the condition in question to be an important public health problem, an effective treatment to be available that might be applied in the latent phase of disease, a suitable test to be available that is acceptable to the population, and cost-benefit to be favorable. ${ }^{6}$ Originally intended to be applied to newborn screening, these same criteria have been adapted to the assessment of population screening for risk of adult-onset conditions. ${ }^{4}$ In the case of carrier screening, the targeted diseases have generally been severe, with limited or no intervention available, and the intended goal is decreasing the incidence of such diseases. However, recent expansion of such screening has included diseases that are less severe and for which treatment or prevention is available. ${ }^{7}$

Studies aimed at assessing risk alleles in populations must also deal with interpreting variants with limited support for pathogenicity. The curation of genetic variants has recently received attention from medical societies, including the $\mathrm{ACMG} .{ }^{8}$ Factors considered by the ACMG and other groups include type of variant, support in the literature, number of cases and controls with the variant and odds ratio, similarity to known pathogenic variants, results of prediction algorithms, and functional studies (Supplementary Figure S1 online). A recent study that performed whole-exome sequencing on 6,517 European and African Americans suggested that $45 \%$ of individuals carry at least one mutation in genes included on newborn screens. ${ }^{9}$

We recently sequenced $128 \mathrm{AJ}$ individuals (The Ashkenazi Genome Consortium data set, or "TAGC dataset") and established a list of previously known and new mutations. ${ }^{10}$ Here we address the pathogenicity of the newly identified mutations and the clinical utility of screening for them.

\section{MATERIALS AND METHODS}

DNA samples were collected from 128 individuals who were disease-free and were verified by principal component analysis to have AJ origin, as we recently described. ${ }^{10}$ DNA sequencing was performed by complete genomics (CG) and annotated using the reference genome version hg19.10

ClinVar and OMIM, publicly available genetic databases that catalog genotypes and phenotypes, were scanned via a custom web crawler to identify variants associated with phenotypes. ${ }^{11}$ Of the 2,434 variants that were identified from sequencing 128 AJ individuals, 80 variants were reported by ClinVar or OMIM as "pathogenic" or "possibly pathogenic." These variants were curated manually by at least two independent reviewers through literature review and assigned a score based on the ACMG scoring system. An additional 56 variants were scored as "pathogenic, very strong" using the ACMG system. Factors considered were number of patients, number of controls, association with other mutations, and functional studies (Supplementary Table S1 online). ${ }^{8}$ Those determined to be pathogenic or likely pathogenic were further reviewed for individual clinical utility and frequency of the variant and then confirmed for their presence in the AJ population using orthogonal whole genome (315 subjects) or whole exome Illumina sequencing (640 subjects).

Calculations of expected prevalence were performed for each variant based on Hardy-Weinberg equilibrium using allele frequencies for our data and carrier frequencies for variants found in the literature (Supplementary Table S2 online). If available, literature frequencies were used as they should be based on a larger number of samples, but for new variants the frequencies from our study were used. However, if the rare mutations had other more common mutations for the same disease, or if they were well-established in the literature in the Ashkenazi population, then they were not removed from the panel. Note that this method of estimation does not include consideration of prevalent Jewish/non-Jewish marriages, which would not impact allele frequencies in the current adult population but would lessen the impact to offspring of one non-Jewish parent.

We assessed the clinical utility of screening for each of the diseases. Factors including opportunity to provide reproductive counseling, diagnosis, early detection of disease, and the impact of early intervention on outcomes were all considered in our analysis for inclusion on the panel. Factors pertaining to personal utility, such as increasing knowledge and control, were also considered. Diseases considered to be benign biochemical traits or lacking clinical utility were removed from the panel. Mutations based on 
PubMed curation that we reported in previous reviews $s^{12,13}$ and on lists from other laboratories that offer AJ carrier screening were assessed for clinical validity and clinical utility and then added if they met the same criteria as the variants identified from the sequenced genomes. The different Ashkenazi screening panels currently available are compared in Supplementary Table S3 online, along with the ACMG and American College of Obstetricians and Gynecologists recommendations.

The impact of the additional mutations in the expanded panel was calculated by comparison to the other panels (Supplementary Table S2 online). For new mutations being added, the number of impacted individuals was calculated based on the Hardy-Weinberg equations. To guarantee that our estimate is conservative, we did not consider mutations with frequency higher than $2 \%$ for dominant mutations and $10 \%$ for recessive ones, unless they were well-established conditions such as congenital adrenal hyperplasia and familial Mediterranean fever. For genes harboring multiple mutations, we assumed that their effects are independent, and that the effects of different genes are independent.

\section{RESULTS}

The final panel included conditions that confer early age of onset risks to offspring and afford the opportunity for reproductive genetic counseling, as well as conditions tested for in the presymptomatic period that inform personal risk of future disease (Table 1 and Supplementary Table S2 online). The 22 conditions that appear on at least one AJ panel currently being offered are all included on this panel. An additional 60 conditions were included based on the criteria of a predicted frequency of at least 1 in 60,000 and clinical utility for the patient, resulting in a total of 163 mutations for 103 conditions. The panel included 76 autosomal recessive, 24 autosomal dominant, and $3 \mathrm{X}$-linked disorders.

\section{Categories of disorders}

Many conditions that fall into the category of presymptomatic screening confer future risk of cancer, such as BRCA1/2 and mismatch repair gene testing, but this group also includes diseases such as obesity and enhanced S cone dystrophy. Diseases that inform primary risk to offspring are mostly autosomal recessive and range from very severe conditions, such as Cockayne syndrome and Leigh syndrome, to milder conditions, such as hyperoxaluria and icthyosis. Genetic testing may be diagnostic for affected children. Some of the dominant conditions, such as Brugada syndrome, adult-onset diabetes, and hypophosphatemic nephrolithiasis/osteoporosis 2, have variable expressivity, and a screening test would be diagnostic and may offer the opportunity for early diagnosis and intervention in affected offspring. There are also some X-linked conditions for which clinical utility may be offered in a variety of ways. FMR1 premutation testing will identify risk of fragile $\mathrm{X}$ syndrome in offspring as well as risks of fragile $\mathrm{X}$-associated tremor/ataxia syndrome and premature ovarian failure. ${ }^{14}$ Other X-linked risks of premature ovarian failure may also be identified.

\section{Clinical utility}

This panel was designed to include highly penetrant Mendelian disorders for which clinical utility may be derived from testing. Therefore, some conditions were removed from our clinical panel if they did not provide clinical utility. Cystathianuria and pentosuria are benign biochemical traits and therefore were not included. Cryptorchidism, a readily apparent and correctable trait, and familial candidiasis, typically quite mild for mutation carriers, were removed due to lack of clinical utility. Warfarin resistance was removed due to the lack of demonstrable clinical utility. The ACMG has recommended against populationbased screening for warfarin resistance due the current paucity of data supporting such screening. ${ }^{15}$ Owing to uncertain penetrance, $A P O E$ was removed.

Some previously reported conditions and variants that were not detected in the TAGC dataset were added to our panel. The c. $1716+1 \mathrm{G}>\mathrm{A}$ variant in the F11 gene causing factor XI deficiency is considered to be an AJ mutation. ${ }^{16}$ Two galactosemia variants in the GALT gene were added, Q188R and K285N, due to their high frequency in Eastern European populations. ${ }^{17}$ The E372X variant in the $B C K D H B$ gene, which causes maple syrup urine disease, was also added due to its high frequency in the AJ population. ${ }^{18}$ Tyrosinemia was added to our panel because the P261L mutation in the FAH gene is known to be prevalent in the AJ population. ${ }^{19}$ Deletion of exon 7 in the SMN1 gene conferring spinal muscular atrophy was added due to its prevalence in all populations and the recommendation from the ACMG to screen all couples regardless of race or ethnicity. ${ }^{20}$ In addition, the following common mutations for congenital adrenal hyperplasia were added: p.P30L, IVS2-13C $>$ G (IVS-2), p.I172N, p.V281L, p.Q318X, p.R356W, and an 8-bp deletion in exon 3. ${ }^{21}$

\section{Varying risk depending on allelic status}

Some recessive conditions that confer different phenotypes in the monoallelic and biallelic states can be considered to offer information pertaining to personal risk as well as risk to offspring. One example is the GBA gene in which a mutation carrier is at risk of having a child affected with Gaucher disease if two mutations are inherited, and also has a personal risk of developing Parkinson disease. ${ }^{22}$ Another example is the ATM gene in which a mutation carrier has a future risk of cancer as well as the risk of having a child with ataxia telangiectasia if two mutations are inherited. ${ }^{23}$ This screening test may also be diagnostic for recessive conditions in adults that can have atypical presentations or later onset. Examples include cystic fibrosis, ${ }^{24}$ Gaucher disease, ${ }^{25}$ and GJB2-associated hearing loss. ${ }^{26}$ For the newly identified mutations, frequencies may be revised once larger numbers of subjects are tested. Some of the low-frequency mutations may be private to the individuals sequenced and may not be found among other members of the AJ population.

A conservative estimate suggests that screening of all mutations in our expanded panel is expected to detect medically relevant dominant mutations in $28 \%$ of patients (approximately 14,000 per year, assuming 50,000 tests per year). Prenatal 
Table 1 Phenotypes by inheritance pattern

\begin{tabular}{|c|c|c|}
\hline $\begin{array}{l}\text { Autosomal dominant phenotype } \\
(n=24)\end{array}$ & $\begin{array}{l}\text { Bronchiectasis with or without elevated sweat } \\
\text { chloride } 2\end{array}$ & Ichthyosis vulgaris \\
\hline Adrenocortical hyperplasia & Canavan disease & Ichthyosis, congenital, autosomal recessive 1 \\
\hline $\begin{array}{l}\text { Arrhythmogenic right ventricular dysplasia/ } \\
\text { cardiomyopathy }\end{array}$ & Candidiasis, familial, 4 & Joubert syndrome \\
\hline Bone marrow failure, telomere-related, 1 & Carnitine palmitoyltransferase 2 deficiency & Leber congenital amaurosis \\
\hline Breast/ovarian cancer predisposition & Charcot-Marie-Tooth disease, type $1 \mathrm{~A}$ & Leigh syndrome \\
\hline Brugada syndrome 1 & Cockayne syndrome A & $\begin{array}{l}\text { Leukoencephalopathy, brain and spine } \\
\text { involvement, lactate elevation }\end{array}$ \\
\hline Carcinoid tumors/Cowden disease 3 & Combined hyperlipidemia, familial & Mandibuloacral dysplasia \\
\hline Cerebral cavernous malformations & Congenital adrenal hyperplasia & Maple syrup urine disease \\
\hline Colon cancer predisposition & Cryptorchidism & $\begin{array}{l}\text { Megalencephalic leukoencephalopathy with } \\
\text { subcortical cysts }\end{array}$ \\
\hline $\begin{array}{l}\text { Corneal dystrophy, hereditary polymorphous } \\
\text { posterior }\end{array}$ & Cystic fibrosis & Mucolipidosis IV \\
\hline Diabetes mellitus, noninsulin-dependent & Cystinuria & Muscular dystrophy, limb girdle 2L \\
\hline Enhanced S-cone syndrome & Deafness, autosomal recessive $1 \mathrm{~A}$ & Myoadenylate deaminase deficiency, myopathy \\
\hline Familial hypercholesterolemia & Deafness, autosomal recessive $1 \mathrm{~B}$ & Nemaline myopathy \\
\hline Hyperglycinuria & Dihydrolipoamide dehydrogenase deficiency & Niemann-Pick disease, type A \\
\hline Hyperlipoprotenemia, type III & Dihydropyrimidine dehydrogenase deficiency & Niemann-Pick disease, type B \\
\hline Idiopathic torsion dystonia & Dyskeratosis congenita, autosomal recessive, 3 & Phosphoglycerate dehydrogenase deficiency \\
\hline Long QT syndrome 5 & Ehlers-Danlos syndrome, type II & Primary ciliary dyskinesia \\
\hline Lynch syndrome & Factor XI deficiency (PTA) & Protoporphyria, erythropoietic \\
\hline Nephrolithiasis/osteoporosis, hypophosphatemic, 2 & Familial dysautonomia & Refsum disease \\
\hline Obesity & Familial hyperinsulinism & Retinitis pigmentosa 28 \\
\hline Parkinson disease 8 & Familial Mediterranean fever & Retinitis pigmentosa 59 \\
\hline Thyroid carcinoma, familial medullary & Fanconi anemia, complementation group $C$ & Retinitis pigmentosa 62 \\
\hline Timothy syndrome & Fructose intolerance & Smith-Lemli-Opitz syndrome \\
\hline Vitelliform macular dystrophy, adult onset & Fucosyltransferase 6 deficiency & Spinal muscular atrophy 37 \\
\hline von Willebrand disease, type $2 \mathrm{~N}$ & Galactosemia & Stargardt disease 1 \\
\hline Autosomal recessive phenotype $(n=76)$ & Gaucher disease, type 1 & Tay-Sachs disease \\
\hline Alpha thalassemia & Glutathione synthetase deficiency & Thrombotic thrombocytopenic purpura, familial \\
\hline Abetalipoproteinemia & Glycogen storage disease la & Usher syndrome, type $1 \mathrm{~F}$ \\
\hline Acyl CoA dehydrogenase deficiency & Hemochromatosis & Usher syndrome, type $3 \mathrm{~A}$ \\
\hline Albinism oculocutaneous, type II & Hermasky-Pudlak syndrome 3 & Walker-Warburg syndrome \\
\hline Albinism, oculocutaneous, type IB & Homocystinuria & Xeroderma pigmentosum, group $\mathrm{C}$ \\
\hline Amegakaryocytic thrombocytopenia & Hyper IgD syndrome & X-linked phenotype $(n=3)$ \\
\hline Bartter syndrome type 3 & Hyperoxaluria, primary, type 3 & Fragile $X$ syndrome \\
\hline Beta-ureidopropionase deficiency & Hyperprolinemia, type I & Premature ovarian failure $2 \mathrm{~B}$ \\
\hline Bloom syndrome & Hypocholinesterasaemia & Premature ovarian failure 4 \\
\hline
\end{tabular}

Bronchiectasis with or without elevated sweat chloride 1

screening for recessive conditions using our expanded panel is expected to affect $3-4 \%$ of the couples $(1,800$ per year, assuming 50,000 prenatal tests per year).

\section{DISCUSSION}

Through our variant analysis we have identified a number of disease variants that are prevalent in the AJ population. These variants provide additional genotyping targets that can be included in an expanded AJ screening panel. Traditionally, the goal of carrier screening has been to decrease the occurrence of severe, untreatable genetic disorders, as evidenced in the case of Tay-Sachs. However, with our increasing ability to identify prevalent disease variants in certain populations, we need to reframe our goals of population screening and genetic counseling.

In assessing the utility of a genetic screening test, clinical endpoints such as reduction in morbidity and mortality have traditionally been used. However, there is a move toward broadening 
this definition of clinical utility to include informational, psychological, and social benefits of undergoing a genetic test. ${ }^{27}$ Also referred to as personal utility, learning one's genetic carrier status or future risk of disease can alleviate anxiety, afford the opportunity for future life planning, and satisfy a need for information, which are all valuable benefits of genetic testing irrespective of clinical use or health outcomes. ${ }^{27}$ For example, in individuals who chose to undergo susceptibility testing for Alzheimer disease, a disease for which there is no proven cure or prevention, information-seeking was an important motivator for pursuing genetic testing, as were logistical and altruistic factors, such as future planning, preparing family members, and contributing to research, emphasizing the importance of considering these other endpoints as measures of the utility of genetic testing.

Gaucher disease serves as a paradigm for conditions that fall outside of the traditional screening criteria but are now part of many AJ panels. Several professional organizations have recommended against AJ population screening for Gaucher disease due to poor genotype/phenotype correlations and lack of data on the efficacy of treatment for mild disease. ${ }^{28}$ Screening for type 1 Gaucher disease, caused by the p.N370S mutation, has been particularly controversial due to its variable expressivity and reduced penetrance. Approximately $90 \%$ of those homozygous for this mutation are mildly affected or completely asymptomatic, yet even symptomatic individuals are diagnosed only at the time of the screening test. ${ }^{7}$ Conditions that do not have a clear-cut genotype/phenotype correlation present challenges for genetic counseling and prenatal decision-making. In addition, for conditions with reduced penetrance, carrier screening may actually uncover an unexpected diagnosis. ${ }^{7}$ An analysis of the Israeli screening program for type 1 Gaucher disease between 1994 and 2005 revealed that most couples did not terminate pregnancies predicted to have mild or asymptomatic disease. In addition, termination was significantly less likely if the couple received genetic counseling or consulted with a Gaucher disease specialist, emphasizing the importance of genetic counseling as an integral part of any screening program.

Nonsyndromic deafness is another condition that demonstrates a limited number of $\mathrm{AJ}$ mutations, but for which the clinical utility of screening may be minimal. It has been demonstrated that two mutations in the GJB2 gene yield a carrier frequency of $4.76 \%$ among $\mathrm{AJ}$ individuals. ${ }^{29}$ Although this frequency is similar to that of Gaucher disease and Tay-Sachs, GJB2 screening is not routinely offered. The identification of carrier risk for hearing loss would aid in the early diagnosis of an affected child, and could identify cases that might be missed by newborn screening - especially because hearing loss may not be present at birth. Early diagnosis can also facilitate early intervention, which has been shown to be beneficial for language acquisition and learning among children who are deaf or hard of hearing. ${ }^{29}$ Familial Mediterranean fever is another condition that falls outside of traditional population screening criteria, but for which carrier screening is widely available..$^{30}$ Because the symptoms of familial Mediterranean fever are generally not life-threatening, and because more than $90 \%$ of patients respond to colchicine treatment, familial Mediterranean fever has generally not been recommended for population-based carrier screening. However, like nonsyndromic hearing loss, there are significant benefits from screening for early diagnosis and treatment that has been shown to be beneficial. ${ }^{31}$ Nonclassical 21-hydroxylase deficiency, a mild form of congenital adrenal hyperplasia, is a condition with variable expressivity and an estimated prevalence of $1 / 100$ in the general population, making it the most common autosomal recessive condition. In the AJ population, the frequency is estimated to be even higher $(1 / 27))^{32}$ Partial 21-hydroxylase deficiency can lead to precocious puberty, cystic acne, and advanced bone age. Affected males usually do not exhibit symptoms, but they may have oligospermia and reduced fertility, whereas women more commonly suffer from menstrual and gonadal dysfunction that can lead to infertility. In both males and females, these symptoms may be reversed with hormonal treatment. Population genetic screening of this frequent yet largely undiagnosed condition can identify affected individuals and offer the opportunity for treatment. ${ }^{33}$

One advantage to applying predisposition screening to a particular group is that ethnicity-based screening exhibits lower rates of variants of uncertain significance, favorably impacting clinical utility and cost-effectiveness. Expanded populationbased BRCA1/2 screening has been shown to be cost-effective in the $\mathrm{AJ}$ population, because screening would lessen the risk of ovarian cancer. ${ }^{5}$ As in the case of $B R C A 1 / 2$ screening, risk assessment for other cancer syndromes, such as Lynch syndrome and Cowden syndrome, provides the opportunity for early detection and intervention to reduce, prevent, or diagnose cancers early. Despite discussion about universal screening for Lynch syndrome among colon cancer patients, no such similar discussion of AJ population screening has taken place. ${ }^{34}$ For other conditions for which there is no intervention available to decrease risk, such as Parkinson disease and various types of deafness, there may still be some utility in gaining the knowledge of one's risk for future life planning as well as informing risks for family members.

Opinions conflict about the benefits of expanding carrier screening for the AJ population. Some worry that the number of diseases on the panel precludes the ability to provide adequate counseling to ensure truly informed consent. Others have concerns about increasing anxiety in individuals who are screened for a multitude of conditions and that, given the low frequency of many of these conditions, this harm outweighs the potential benefits. ${ }^{35}$ Modifications to the standard informed consent process for single gene tests should be considered when screening for multiple conditions. Because providing a detailed discussion about each disease on the panel is not feasible, new models of consent will be needed to explain risks and benefits effectively and to assess patient understanding. Healthcare professionals beyond genetic counselors and medical geneticists should be trained to convey this information to meet the growing demand. ${ }^{36}$ In a study of pregnant women and their partners regarding 
informed consent for genetic carrier screening, one significant finding was confusion about the purpose of genetic screening. Individuals associated this screening with gaining information about their offspring and not about themselves. ${ }^{37}$ Nonetheless, when explained that personal risks could also be identified, most individuals in another study indicated a preference for screening. ${ }^{38}$ Because the diseases on this panel impart both reproductive and personal risks, with some mutations conferring both, it will be important to emphasize the different types of information that may be gleaned from genetic screening. Patients vary in their preferences of a generic consent model, in which general principles of screening are explained with the details of each disease discussed only if the patient tests positive, versus a comprehensive consent model in which details about each disease being screened for are given prior to testing. This variability calls for a more personalized approach to the consent process to meet the patient's needs. Of course, with an extensive screening panel, even applying the comprehensive model of consent will have its limitations due to the sheer amount of information that would need to be conveyed for all the diseases. In addition to a more personalized approach to the consent process, it would be beneficial to allow individuals to choose what they would like to be tested for due to the broad range of diseases offered on our panel and the varying implications for both personal and reproductive risk,. Such an approach will enhance autonomy for the patient by respecting his/her right not to know. ${ }^{39}$

\section{SUPPLEMENTARY MATERIAL}

Supplementary material is linked to the online version of the paper at http://www.nature.com/gim

\section{ACKNOWLEDGMENTS}

We thank Eli Balshan and Michael Groden for assistance with annotation. We acknowledge financial support from the Human Frontier Science Program (S.C.); NIH research grants AG042188 (G.A.); DK62429, DK062422, and DK092235 (J.H.C.); NS050487 and NS060113 (L.N.C.); AG021654 and AG027734 (N.B.); MH089964, MH095458, and MH084098 (T.L.), and CA121852 (computational infrastructure, IPe'er); NSF research grants 08929882 and 0845677 (IPe'er); the Rachel and Louis Rudin Foundation (H.O.); the North Shore-LIJ Health System Foundation (T.L.); the New York Crohn's Foundation (IPeter); the Parkinson's Disease Foundation (L.N.C.); the Sharon Levine Corzine Cancer Research Fund (K.O.); and the Andrew Sabin Family Research Fund (K.O.) and wholeexome sequence data from the T2D-GENES Consortium.

\section{DISCLOSURE}

The authors declare no conflict of interest.

\section{REFERENCES}

1. Kaback MM. Population-based genetic screening for reproductive counseling: the Tay-Sachs disease model. Eur J Pediatr 2000;159(suppl 3):S192-S195.

2. Lazarin GA, Haque IS, Nazareth $S$, et al. An empirical estimate of carrier frequencies for $400+$ causal Mendelian variants: results from an ethnically diverse clinical sample of 23,453 individuals. Genet Med 2013;15:178-186.

3. Khoury MJ, Gwinn M, Yoon PW, Dowling N, Moore CA, Bradley L. The continuum of translation research in genomic medicine: how can we accelerate the appropriate integration of human genome discoveries into health care and disease prevention? Genet Med 2007;9:665-674.

4. Burke W, Coughlin SS, Lee NC, Weed DL, Khoury MJ. Application of population screening principles to genetic screening for adult-onset conditions. Genet Test 2001;5:201-211.

5. Rubinstein WS, Jiang H, Dellefave L, Rademaker AW. Cost-effectiveness of population-based BRCA1/2 testing and ovarian cancer prevention for Ashkenazi Jews: a call for dialogue. Genet Med 2009;11:629-639.

6. Wilson JMG, Jungner G. Principles and Practice of Screening for Disease. 1968. http://whqlibdoc.who.int/php/WHO_PHP_34.pdf. Accessed 17 March, 2014.

7. Zuckerman S, Lahad A, Shmueli A, et al. Carrier screening for Gaucher disease: lessons for low-penetrance, treatable diseases. JAMA 2007;298:1281-1290.

8. Richards S, Aziz N, Bale S, et al.; ACMG Laboratory Quality Assurance Committee. Standards and guidelines for the interpretation of sequence variants: a joint consensus recommendation of the American College of Medical Genetics and Genomics and the Association for Molecular Pathology. Genet Med 2015; 17:405-424.

9. Tabor HK, Auer PL, Jamal SM, et al.; NHLBI Exome Sequencing Project. Pathogenic variants for Mendelian and complex traits in exomes of 6,517 European and African Americans: implications for the return of incidental results. Am J Hum Genet 2014;95:183-193.

10. Carmi S, Hui KY, Kochav E, et al. Sequencing an Ashkenazi reference panel supports population-targeted personal genomics and illuminates Jewish and European origins. Nat Commun 2014;5:4835.

11. National Center for Biotechnology Information. What Is ClinVar? https://www. ncbi.nlm.nih.gov/clinvar/intro/. Accessed 17 March, 2014.

12. Ostrer H. A genetic profile of contemporary Jewish populations. Nat Rev Genet 2001;2:891-898.

13. Ostrer H, Skorecki K. The population genetics of the Jewish people. Hum Genet 2013:132:119-127.

14. Saul RA, Tarleton JC. FMR-1-Related Disorders. 1998. http://www.ncbi.nlm.nih. gov/books/NBK1384/.

15. Flockhart DA, O'Kane D, Williams MS, et al.; ACMG Working Group on Pharmacogenetic Testing of CYP2C9, VKORC1 Alleles for Warfarin Use. Pharmacogenetic testing of CYP2C9 and VKORC1 alleles for warfarin. Genet Med 2008;10:139-150.

16. Peretz H, Salomon O, Mor-Cohen $\mathrm{R}$, et al. Type I mutation in the F11 gene is a third ancestral mutation which causes factor XI deficiency in Ashkenazi Jews. J Thromb Haemost 2013;11:724-730.

17. Tyfield L, Reichardt J, Fridovich-Keil J, et al. Classical galactosemia and mutations at the galactose-1-phosphate uridyl transferase (GALT) gene. Hum Mutat 1999;13:417-430.

18. Edelmann L, Wasserstein MP, Kornreich R, Sansaricq C, Snyderman SE, Diaz GA. Maple syrup urine disease: identification and carrier-frequency determination of a novel founder mutation in the Ashkenazi Jewish population. Am J Hum Genet 2001;69:863-868.

19. Elpeleg ON, Shaag A, Holme E, et al. Mutation analysis of the FAH gene in Israeli patients with tyrosinemia type I. Hum Mutat 2002;19:80-81. Accessed 17 March, 2014.

20. Prior TW; Professional Practice and Guidelines Committee. Carrier screening for spinal muscular atrophy. Genet Med 2008;10:840-842. Accessed 17 March, 2014.

21. Wilson RC, Nimkarn S, Dumic M, et al. Ethnic-specific distribution of mutations in 716 patients with congenital adrenal hyperplasia owing to 21-hydroxylase deficiency. Mol Genet Metab 2007;90:414-421.

22. Becker JG, Pastores GM, Di Rocco A, Ferraris M, Graber JJ, Sathe S. Parkinson's disease in patients and obligate carriers of Gaucher disease. Parkinsonism Relat Disord 2013;19:129-131.

23. Byrnes GB, Southey MC, Hopper JL. Are the so-called low penetrance breast cancer genes, ATM, BRIP1, PALB2 and CHEK2, high risk for women with strong family histories? Breast Cancer Res 2008;10:208.

24. Boyle MP. Nonclassic cystic fibrosis and CFTR-related diseases. Curr Opin Pulm Med 2003;9:498-503.

25. Jain VV, Yelwatkar S. Unusual presentation of adult Gaucher's disease: A long and difficult road to diagnosis. Indian J Endocrinol Metab 2011;15: 224-226.

26. Tekin M, Duman T, Boğoçlu G, Incesulu A, Cin S, Akar N. Moderate hearing loss and pseudodominant inheritance due to L90P/35delG mutations in the GJB2 (connexin 26) gene. Genet Couns 2003;14:379-386.

27. Grosse SD, Khoury MJ. What is the clinical utility of genetic testing? Genet Med 2006;8:448-450.

28. Gaucher disease. Current issues in diagnosis and treatment. NIH Technology Assessment Panel on Gaucher Disease. JAMA 1996;275:548-553. 


\section{ORIGINAL RESEARCH ARTICLE}

29. Morell RJ, Kim HJ, Hood LJ, et al. Mutations in the connexin 26 gene (GJB2) among Ashkenazi Jews with nonsyndromic recessive deafness. N Engl J Med 1998;339:1500-1505.

30. GeneDx. Familial Mediterranean Fever. http://www.genedx.com/test-catalog/ disorders/familial-mediterranean-fever/.

31. Padeh S, Livneh A, Pras E, et al. Familial Mediterranean Fever in the first two years of life: a unique phenotype of disease in evolution. J Pediatr 2010;156: 985-989. Accessed 17 March, 2014.

32. Speiser PW, Dupont B, Rubinstein P, Piazza A, Kastelan A, New MI. High frequency of nonclassical steroid 21-hydroxylase deficiency. Am J Hum Genet 1985;37:650-667

33. Strom CM, Crossley B, Redman JB, et al. Molecular screening for diseases frequent in Ashkenazi Jews: lessons learned from more than 100,000 tests performed in a commercial laboratory. Genet Med 2004;6: 145-152.
34. Hampel H, Frankel WL, Martin E, et al. Feasibility of screening for Lynch syndrome among patients with colorectal cancer. J Clin Oncol 2008;26: 5783-5788.

35. Dolgin, E. Jewish genetic screening grows despite questions about breadth. Nat Med 2011;17:639.

36. Ormond KE, Wheeler MT, Hudgins L, et al. Challenges in the clinical application of whole-genome sequencing. Lancet 2010;375:1749-1751.

37. Ormond KE, Iris M, Banuvar S, Minogue J, Annas GJ, Elias S. What do patients prefer: informed consent models for genetic carrier testing. J Genet Couns 2007; 16:539-550.

38. Hathaway F, Burns E, Ostrer H. Consumers' desire towards current and prospective reproductive genetic testing. J Genet Couns 2009;18:137-146.

39. Andorno R. The right not to know: an autonomy based approach. J Med Ethics 2004:30:435-9. 